\title{
Biodegradation kinetics of microcystins-LR crude extract by Lysinibacillus boronitolerans strain CQ5
}

\author{
Rongyan Shen ${ }^{1} \cdot$ Zehui Chen $^{1} \cdot$ Xiaona Dong ${ }^{1} \cdot$ Hongchi Shen ${ }^{1} \cdot$ Peng Su ${ }^{1} \cdot$ Linqiang Mao $^{1} \cdot$ Wenyi Zhang $^{1}$
}

Received: 30 May 2019 / Accepted: 8 August 2019 / Published online: 27 August 2019

(C) Università degli studi di Milano 2019

\begin{abstract}
Purpose As the most common variant of microcystins (MCs), microcystin-LR (MCLR) is a kind of toxins produced by some species of harmful cyanobacteria and more and more attention has been paid to it. Biodegradation has been extensively investigated and recognized to be a cost-efficient and environmentally benign method for MC clean-up. In order to further research the growth characteristics of strain and the biodegradation characteristics of MCLR, it is necessary to use the dynamic mathematical models as powerful and useful tools.

Methods In this study, strain CQ5 was screened and identified by morphological observation, physiological and biochemical tests, and 16S rDNA sequence analysis. The kinetic models of cell growth and MCLR degradation were established with the Gompertz model and revised Monod kinetic model.

Results The results showed that strain CQ5 had the closest phylogenetic similarity to Lysinibacillus boronitolerans (T-10a, AB199591) in the phylogenetic tree, with 99\% bootstrap support. Strain CQ5 could utilize MCLR as the carbon and nitrogen source for growth. When the initial $\mathrm{pH}$ value was 7 and the inoculation amount was 3\%, strain CQ5 grew well in MSM, in which the MCLR crude extract was used as the carbon and nitrogen source of strain CQ5. Within $244 \mathrm{~h}$, the MCLR concentration changed from 14.12 to $1.57 \mu \mathrm{g} / \mathrm{L}$ and its degradation rate could reach $88.88 \%$. The growth curve fitted with the Gompertz growth model $\left(N_{\mathrm{t}}=1.3119^{*} \exp \left(-0.1237^{*} \exp (-6.6341 t)\right), R^{2}>0.99\right)$. The process of MCLR degradation agreed with the first-order reaction kinetic equation $\left(\ln S=2.64764-0.01537 t, R^{2}>0.99\right)$. The linkage relationship between MCLR concentration, cell density, and MCLR degradation rate was consistent with the revised Monod equation $\left(V=0.342 S, R^{2}>0.97\right)$ at low substrate concentration, where $V_{\max } / K_{s}$ was 0.342 . The dynamic relationship in which strain CQ5 degraded MCLR and used it as the carbon and nitrogen source to promote its own growth could be explained by the equation $S=14.12 e^{-0.342 N t}(N=1.08)$.

Conclusion The growth of strain CQ5 and MCLR concentration in degradation system could be simulated and predicted by the dynamic mathematical models in this study. And the predicted results were very consistent. These results could provide theoretical reference for studying the mechanism of MCLR biodegradation and promote the engineering application of strain CQ5.
\end{abstract}

Keywords Microcystins-LR (MCLR) $\cdot$ Biodegradation kinetics $\cdot$ Gompertz model $\cdot$ First-order reaction equation $\cdot$ Monod model

\section{Introduction}

Harmful cyanobacterial blooms in natural water bodies have become a worldwide environmental problem as a consequence of eutrophication and climate change (Dziga et al. 2013; Li and Pan 2014). Microcystins (MCs) are a family of cyclic heptapeptide toxins produced by some species of

Wenyi Zhang

zwy@cczu.edu.cn

1 School of Environmental and Safety Engineering, Changzhou University, Changzhou 213164, China harmful bloom-forming cyanobacteria. More than 85 variants of MCs have been identified, of which microcystin-LR (MCLR) is the most common and studied variant (Vareli et al. 2013; Rastogi et al. 2014; Zhu et al. 2016).

Numerous studies have been issued towards the removal of MCs from natural waters due to their hepatotoxicity and potential carcinogenic activity and even lethal effects these toxins pose to ecosystems and public health (Eleuterio and Batista 2010; Wu et al. 2010; Zhu et al. 2016). As the main approach for MC elimination from aquatic habitats, biodegradation has been extensively investigated and recognized to be a cost-efficient and environmentally benign clean-up strategy for MCs (Ho et al. 2012; Zhao et al. 2013; Li et al. 2014). 
It has been confirmed that MCs can be efficiently degraded by a range of heterotrophic bacteria indigenous to diverse ecosystems (Lam et al. 1995; Edwards et al. 2008; Ho et al. 2012; Ramani et al. 2012; Song et al. 2014). This implies that MC-degrading bacteria are relatively prevalent in natural environments. Over recent decades, many bacterial strains capable of degrading MCs have been isolated from the UK, Australia, Japan, and Germany (Hall et al. 2007; Bourne et al. 2010; Shimizu et al. 2011), the Torrens Lake in South Australia (Ho et al. 2007), Forfar Loch and the River Carron in the Scotland (Manage et al. 2009), Lake Taihu and Lake Chaohu in China (Wang et al. 2014; Yan et al. 2014; Yang et al. 2014; You et al. 2014), etc. A strain of Sphingopyxis sp. (SW1) was isolated and it had better degradation effect of MCLR and microcystin-RR (Zhao et al. 2013). Burkholderia vietnamiensis strain $\mathrm{C} 09 \mathrm{~V}$ was screened and its MCLR degradation rate was $97.6 \%$ within $48 \mathrm{~h}$ (Wang et al. 2014). By the fitting first-order kinetic equation and the characterization of Fourier transform infrared spectroscopy (FITR) and scanning electron microscope (SEM), it was found that strain C09V could degrade and use MCLR by a series of biochemical degradation in vivo and its cell surface (Wang et al. 2014). Different microorganisms may have different degradation mechanisms of MCs (Yan et al. 2014). So it is of great significance to enrich and develop the biodegradation mechanism of MCs for the construction of efficient gene engineering of bacteria.

The dynamic mathematical models are powerful tools to simulate the behavior of microbial dynamics and the evolution of system structure. And they are also the key tools to link the phenomenon of microbial dynamics with the macro index of large-scale process operation (Chen and Chai 2006). So far, the use of mathematical models of microbial dynamics has been predominantly used in the field of relevant research (Spanjers et al. 1999; Rongsayamanont et al. 2010; Oliveira et al. 2011; Leyva-Díaz et al. 2017). The Gompertz model was originally proposed by B. Gompertz, a British statisticians and mathematicians, to be used as the animal population growth model and to describe the law of extinction of the population (Jia et al. 2009). Gompertz curve was usually used to describe the process of the development of things from germination and growth to saturation. In reality, there are many phenomena that conform to the Gompertz curve, such as the growth of industrial production, the life cycle of the product, and the population growth in a certain period of time. So the curve was widely used in the study on the trend of phenomena (Jia et al. 2009). As an empirical equation of describing the law of microbial growth, the Monod model has also been widely applied in engineering practice (Zhang 2001; Chen and Chai 2005; Zhang et al. 2011). The classical Monod equation had some limitations when the microbial concentration was high and the substrate concentration was low. Therefore, the substrate-revised Monod equation was proposed (Wang et al. 2013). Through theoretical analysis and simulation calculation, it was proven that the modified equation could well describe the microbial growth law under the conditions of high cell concentration and low substrate concentration (Wang et al. 2013).

In this study, Lysinibacillus boronitolerans strain CQ5 was isolated and identified from the sediments sampled from Lake Taihu. The dynamic model of MCLR degradation was established. And the dynamic relationship was investigated between the MCLR concentration, the degradation rate, and the growth of strain CQ5 in the degradation process. The mechanisms of CQ5 degrading MCLR were deduced. These results will provide the theoretical references for the engineering application of MCLR degradation bacteria.

\section{Material and methods}

\section{Chemicals and main instruments}

The MCLR standard was purchased from the Shanghai Enzyme-linked Biotechnology Co. Ltd. Methanol was of high-performance liquid chromatography grade. All other chemicals and solvents were of analytical reagent grade. Microcystins enzyme-linked immunosorbent assay kit, bacterial genomic DNA extraction kit (Ezup column), and T vector recombinant colony PCR assay kit were purchased from the Shanghai Enzyme-linked Biotechnology Co. Ltd. Multiskan Mk3 Elisa and TGL-16C DNA amplification instrument were from the American Thermo Fisher Scientific and Technology Co. Ltd. $752 \mathrm{~N}$ ultraviolet spectrophotometer was from the Shanghai Precision and Scientific Instrument Co. Ltd. and PHS-3C pH meter was from the Shanghai Precision and Scientific Instrument Co. Ltd. Olympus_BX43 researchlevel microscope was from the Shanghai Fulai Optics Technology Co. Ltd. and SC810 gel imaging system was from the Shanghai Chuangmeng Biological Technology Co. Ltd.

\section{Cyanobacterial samples}

Dehydrated algal mud was collected from the blue algal station situated in Baidu Port of Lake Taihu. Then it was dried. Dried algae were milled by $400 \mathrm{rpm}$ for $2 \mathrm{~h}$ and meshed by 60 mesh. The algal powder was kept at $-18^{\circ} \mathrm{C}$ from light.

\section{Media}

The mineral salt medium (MSM) was used as the basal medium for isolation and degradation studies. It was composed of $1.6 \mathrm{~g} \mathrm{~K}_{2} \mathrm{HPO}_{4}, 0.4 \mathrm{~g} \mathrm{KH}_{2} \mathrm{PO}_{4}, 0.2 \mathrm{~g} \mathrm{MgSO}_{4} \cdot 7 \mathrm{H}_{2} \mathrm{O}, 0.5 \mathrm{~g}$ $\mathrm{NaCl}, 20 \mathrm{mg} \mathrm{CaCl}$, and $2.3 \mathrm{mg} \mathrm{FeCl} \cdot 6 \mathrm{H}_{2} \mathrm{O}$ per $1000-\mathrm{mL}$ water. The crude extract of MCLR was extracted and used as a carbon and nitrogen source in the MCLR-containing MSM. 
Then the $\mathrm{pH}$ was regulated to 7.0. The solid medium was prepared by adding $1.5 \%(\mathrm{w} / \mathrm{v})$ agar to the liquid medium. All of the media were sterilized by autoclaving at $121^{\circ} \mathrm{C}$ for $30 \mathrm{~min}$.

\section{Bacterial isolation and identification}

Surface sediment was sampled from Lake Taihu during the algal outbreak period (July 15, 2017). The slurry of sediment was obtained in the shaker at $140 \mathrm{rpm}$ for $4 \mathrm{~h}$. Then $10-\mathrm{mL}$ slurry was transferred to 50-mL MCLR-containing MSM and continuously domesticated 3 times. In the above process, the concentrations of MCLR were gradually increased. Then a tenfold serial dilution of the domesticated bacterial liquid was spread on MSM agar plates containing MCLR and incubated at $30{ }^{\circ} \mathrm{C}$ for 7 days. Individual colonies were tested for the abilities to degrade MCLR in MSM. These colonies that degraded MCLR were re-spread on MCLR-containing MSM plates until pure cultures were obtained. One isolate with high MCLR-degrading capacity was isolated and named CQ5. The cell morphology of strain CQ5 was observed by the scanning electron microscope and gram stains were analyzed by the light microscope.

The 16S rDNA of strain CQ5 was amplified using the universal primers 27F (5'-AGAGTTTGATCCTGGCTCGA$\left.3^{\prime}\right)$ and 1492R (5'-GGYTACCTTGTTACGACTT-3'). The amplified product was analyzed by gel electrophoresis on a $1 \%$ agarose gel and purified sequenced by the Shanghai Shenggong Biological Engineering Technology Service Co. Ltd. A sequence similarity search was conducted by the blastn program in NCBI. The $16 \mathrm{~S}$ rDNA sequences from type strains with high similarity to the study sequence were retrieved from GenBank and aligned with the sequence of strain CQ5 by the ClustalX software. A phylogenetic tree was inferred using a neighbor-joining method in MEGA5.1.

\section{Extraction and detection of MCLR}

In this study, MCLR crude extract was used to regulate the MCLR content in the culture medium during the degradation experiment. The MCLR crude extract was extracted from algal powder by the described method (Rao et al. 2011). The MCLR content was determined by microcystins ELISA kit. The detection limit of MCLR was $0.05-0.80 \mu \mathrm{g} / \mathrm{L}$. The detailed detection step was carried out according to the kit instructions.

\section{MCLR biodegradation}

According to our previous studies, the optimum $\mathrm{pH}$ of strain CQ5 was 7, and the optimum capacity of bacteria was $3 \%$ in the degradation test (Chen et al. 2018). Under the above conditions, the activated CQ5 bacterial solution was inoculated into 30-mL MCLR-containing MSM. And the uninoculated samples were used as the control (uninoculated). Then the samples were cultivated by $140 \mathrm{rpm}$ and cell density of strain was determined every $2 \mathrm{~h}$. The cell density of strain was expressed by the optical density value $\mathrm{OD}_{600}$. Samples were sampled every 1 day and centrifuged by $8000 \mathrm{rpm}$ for $10 \mathrm{~min}$. Then the liquid supernatant was saved for determining the content of MCLR. The MCLR degradation rate was calculated by the equation of $\left(C_{\text {old }}-C_{\text {new }}\right) / C_{\text {old }} \times 100 \%(C$, the concentration of MCLR).

Since the 1990s, many investigations showed that the contents of MCs were from 0.13 to $20 \mu \mathrm{g} / \mathrm{L}$ in Taihu, Poyang Lake, the Yanghe reservoir in Qinhuangdao, and Beijing Guanting Reservoir ( $\mathrm{Mu}$ et al. 2000; Ou et al. 2013; Guo et al. 2016). At the same time, the temperature of Taihu shallow water was about $28-35^{\circ} \mathrm{C}$. So in this degradation test, the initial MCLR concentration and culture temperature were set as $14.12 \mu \mathrm{g} / \mathrm{L}$ and $30^{\circ} \mathrm{C}$, respectively.

\section{Kinetic study}

The Gompertz model was used to describe the growth process of strain CQ5. The Gompertz equation was described by the equation:

$N_{t}=K \times \exp (-a \times \exp (-r t))$

where $N_{t}$ is the cell density of strain at the time of $\mathrm{t}\left(\mathrm{OD}_{600}\right), t$ is the time (h), $K$ is the environmental bearing capability for the strain growth $\left(\mathrm{OD}_{600}\right), r$ is the average growth rate of strain $\left(\mathrm{h}^{-1}\right)$, and $a$ is one parameter related to $K$ and $N_{O}$.

The first-order reaction equation was used to analyze the relationship between the degradation rate of MCLR and the concentration of substrate. The first-order reaction equation was described by the following equation:

$d S / d t=-k S$

Integral was done on both sides of Eq. (2) and the following equation was received:

$\ln S=-k t+\ln S_{0}$

where $S$ is the mass concentration of MCLR in the reaction system at $t(\mu \mathrm{g} / \mathrm{L}), t$ is the degradation time $(\mathrm{h}), k$ is the rate constant of first-order reaction $\left(\mu \mathrm{g} \cdot(\mathrm{L} \cdot 24 \mathrm{~h})^{-1}\right)$, and $S_{0}$ is the initial concentration of MCLR $(\mu \mathrm{g} / \mathrm{L})$.

The general model for Monod was written as

$V=V_{\max }\left(S /\left(K_{s}+S\right)\right)$

The general Monod equation had some limitations when the microbial concentration was high and the substrate concentration was low. Therefore, the substrate-revised Monod equation was proposed (Wang et al. 2013). It was written as: 
$V=\left(V_{\max } / K_{S}\right) S=K_{2} S$

where $K_{s}$ is the saturation constant (the concentration of MCLR, $\mu \mathrm{g} / \mathrm{L}$, the proliferation rate of strain CQ5 $\mu=1$ / $\left.2 \mu_{\max }\right) . V$ is the specific degradation rate of MCLR $(\mu \mathrm{g} /(\mathrm{L}$. $24 \mathrm{~h})$ ). $V_{\max }$ is the maximum specific degradation rate of $\operatorname{MCLR}(\mu \mathrm{g} /(\mathrm{L} \cdot 24 \mathrm{~h})) . S$ is the mass concentration of MCLR $(\mu \mathrm{g} / \mathrm{L}) . K_{2}$ is the specific value of $V_{\max }$ and $K_{s}$. Vexpresses the degradation rate of organic substrate of unit biomass. It could be expressed by the following equation:

$V=-[d S /(d t \cdot N)]=K_{2} S$

where $N$ is the natural growth quantity of strain CQ5 and is expressed by cell density $\mathrm{OD}_{600}$.

\section{Results and discussion}

\section{Isolation and identification of MCLR-degrading bacterium}

Strain CQ5 was isolated from the sediment of Lake Taihu in Jiangsu Province, China. Its colony was round, milky, and transparent on MCLR-containing MSM plates. And its diameter was about 1-4 $\mathrm{mm}$. Its colony became larger at the later stage of culture, and its surface was dry and wrinkled. The edge was slightly irregular and yellow. Its cells were rod-shaped and were gram-positive by light microscope. It could utilize MCLR as the carbon and nitrogen source for growth. To identify this strain, the $16 \mathrm{~S}$ rDNA sequence was amplified and a phylogenetic tree was constructed using this sequence and its similar sequences from type strains (Fig. 1). Deduced are $16 \mathrm{Sr}$ DNA sequences (1419 bp) from type strains obtained from GenBank. Evolutionary analyses were conducted by the neighbor-joining method using the Tamura-Nei substitution model in MEGA5.1. Bootstrap values represented percentages from 1000 replicates of the data, and percentages $>50 \%$ were shown in Fig. 1 . It could be seen that strain CQ5 had the closest phylogenetic similarity to Lysinibacillus boronitolerans (T-10a, AB199591) in this tree, with $99 \%$ bootstrap support.

\section{Degradation characteristics of MCLR by strain CQ5}

When the initial $\mathrm{pH}$ value was 7 and the concentration of MCLR was $14.12 \mu \mathrm{g} / \mathrm{L}$, the activated CQ5 bacterial solution was inoculated into 30-mL MCLR-containing MSM by $3 \%(\mathrm{v} / \mathrm{v})$ inoculation quantity. The uninoculated samples were used as the control. Then they were shake cultured by $140 \mathrm{rpm}$ for 6 days at $30^{\circ} \mathrm{C}$. The MCLR degradation curve was shown in Fig. 2. We could see that MCLR degradation by strain CQ5 was slow at the beginning of the first day $(24 \mathrm{~h})$. The mass concentration of MCLR only decreased by $1.56 \mu \mathrm{g} / \mathrm{L}$. The possible reason might be that strain CQ5 was in the adaptive process of exogenous xenobiotics from the transferred new medium. Strain CQ5 might firstly use a small quantity of protein culture medium from transferred bacterial liquid and nutriment with relatively simple molecular structure from radical and crude nutrient liquid. And oligopeptide structure existed in peptone, whose structure was similar to the molecular structure of MCs. MCs were accidentally degraded by strain CQ5 in the use of peptone. That might ensure the normal growth of strain (Yang et al. 2009; Black 2012). MCLR degradation by strain CQ5 was accelerated the next day $(48 \mathrm{~h})$. The degradation rate was up to $68.45 \%$ ( $72 \mathrm{~h}$, Fig. 2 b). After a large number of MCLR were degraded, the nutrient mediums were insufficient, especially for carbon and nitrogen sources, so the degradation rate of MCLR slowed down on the fourth day (96 h). On the sixth day (144 h), the residual concentration of MCLR was $1.57 \mu \mathrm{g} / \mathrm{L}$ (Fig. 2a) in degradation system and the degradation rate reached $88.88 \%$ (Fig. $2 b)$. But for the control group, MCLR concentration did not obviously change. So the strain CQ5 had a strong capacity of degrading MCLR.
Fig. 1 Phylogenetic analysis of the 16S rDNA sequences from strain CQ5

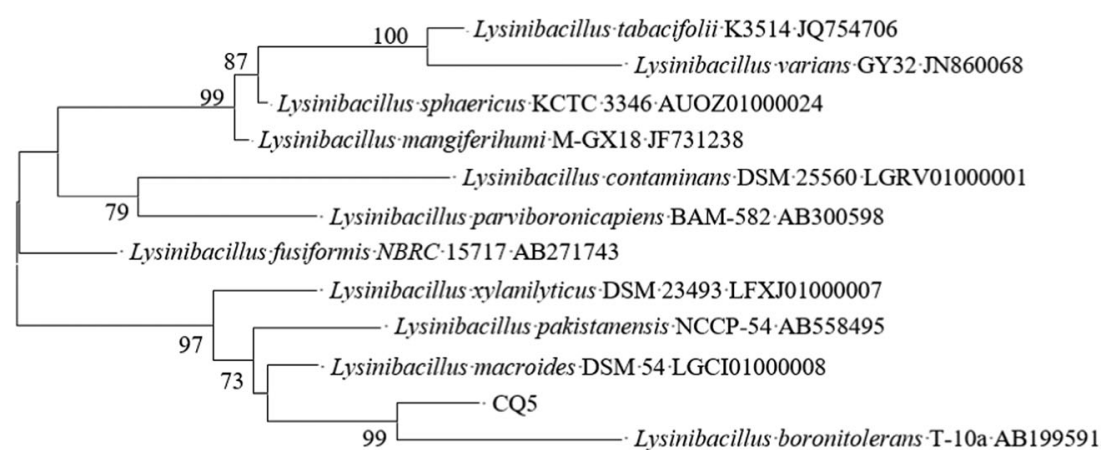

0.002 
Fig. 2 Biodegradation curves of MCLR by strain CQ5. a Changes in MCLR concentrations. $\mathbf{b}$ Changes in MCLR degradation rates

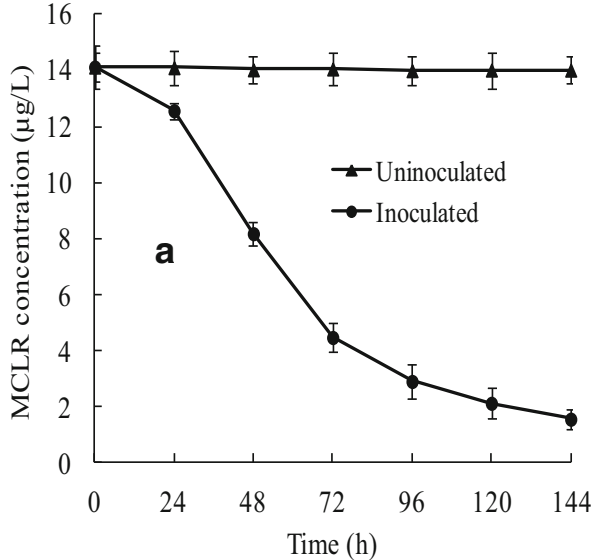

\section{Gompertz model and first-order reaction kinetic analysis}

The degradation curves of MCLR by strain CQ5 and the growth curve of strain CQ5 were shown in Figs. 2a, b and 3, respectively. MCLR degradation in culture medium and the growth of strains simultaneously proceeded along with the lengthened culture time. When the mass concentration of MCLR gradually decreased, strain CQ5 underwent first in a logarithmic growth and then entered into a stable state. The highest cell density of strain CQ5 reached 1.344. At the beginning of the experiment, the crude extracts of MCLR were used to regulate MCLR concentrations in the mineral salt medium. There were impurities to be used as microbial nutrition. So in this study, the cell density of strain was higher than the microbial cell density in the reported MC degradation tests (Xie et al. 2012; Zhao et al. 2012; You et al. 2014). It showed that strain CQ5 could grow well in a complex culture environment and effectively degrade MCLR. It also had certain practical value for degrading MCLR in natural water.

The Gompertz model was often used to describe the S-type growth of population in a limited environment, which was important to study the changes in population dynamics. In this paper, strain CQ5 grew in the MCLR-containing MSM. With

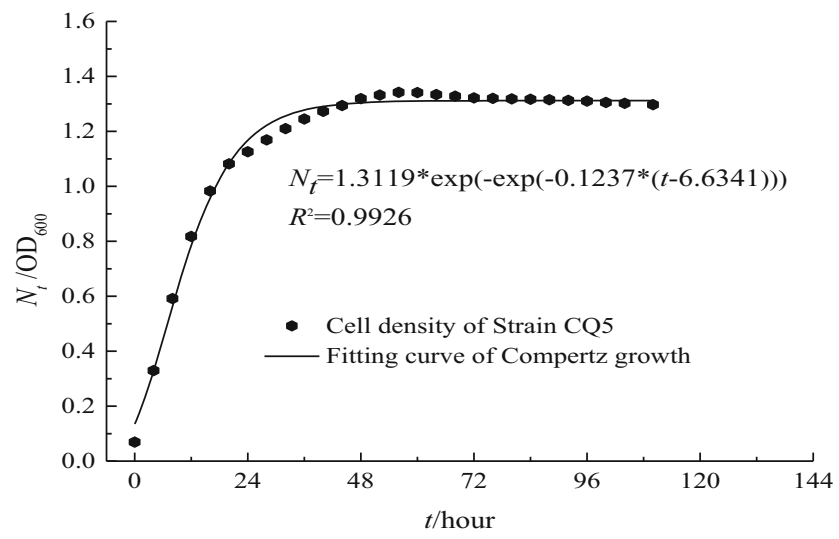

Fig. 3 The Gompertz growth fitting curve of strain CQ5 the growth of strain CQ5, the carbon and nitrogen sources became limited. Therefore, the Gompertz model was used to describe the growth process of strain CQ5.

When the initial cell density of strain CQ5 $\left(N_{0}\right)$ was 0.069 and the maximum cell density $\left(N_{58}\right)$ was 1.344 (Fig. 3), the Gompertz equation curve fitting was carried out between the cell density of strain CQ5 and incubation time by origin 8.5.1, as shown in Fig. 3. And the parameters in the equation were solved. As could be seen from Fig. 3, the growth situation of strain CQ5 could be expressed by the following equation:

$N_{\mathrm{t}}=1.3119 \times \exp (-0.1237 \times \exp (-6.6341 t))$

where $K$ was $1.3119\left(\mathrm{OD}_{600}\right), a$ was $0.1237, r$ was 6.6341 $\left(\mathrm{h}^{-1}\right)$, and the correlation coefficient $\left(R^{2}\right)$ was 0.9926 .

It could be seen that the degradation rate of MCLR by strain CQ5 increased along with the decrease of MCLR concentration in the medium (Fig. 2). So the first-order reaction equation was used to analyze the relationship between the degradation rate of MCLR and the concentration of substrate. And the quantitative relationship between the mass concentration of MCLR and time was studied in the degradation process. The first-order reaction equation was shown by Eq. (2) $(d S / d t=-k S)$.

As the initial concentration of MCLR was $14.12 \mu \mathrm{g} / \mathrm{L}$, the linear fitting was done between $\ln S$ and $T$. The results were shown in Fig. 4.

The first-order reaction kinetics of MCLR degradation by strain CQ5 were expressed by the following equations:

$d S / d t=-0.01537 S$

$\ln S=2.64764-0.01537 t$

where the kinetic reaction rate constant $k$ was 0.01537 . The half-time of $t_{1 / 2}$ was 1.88 days. The correlation coefficient $\left(R^{2}\right)$ was 0.9939 . Then, according to Eqs. (8) and (9), the relationship between the MCLR concentration and the degradation time was further deduced, as shown by the following equation:

$S=e^{2.64764-0.01537 t}$ 


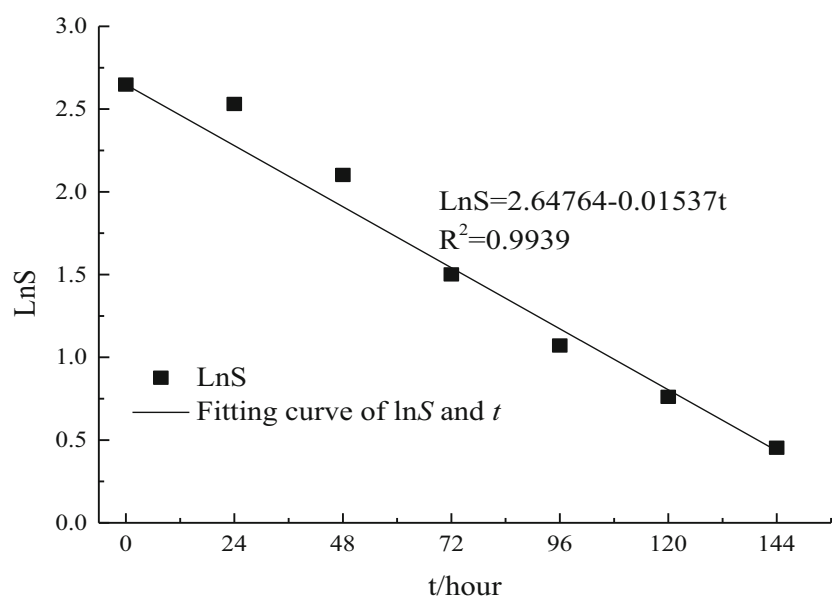

Fig. 4 The degradation kinetic curve of MCLR

\section{Monod model of cell growth of strain CQ5}

In this paper, the degradation test was conducted in the inorganic salt medium in which MCLR crude extract was added as the nitrogen and carbon source. The mass concentration of MCLR was a controlling factor in the growth of strain CQ5. And it was found that the mass concentration of MCLR was also the factors affecting the MCLR degradation rate in the degradation kinetics of MCLR degradation by strain CQ5. It could be inferred that the linkage relationship existed among MCLR mass concentration, cell density of strain CQ5, and the degradation rate of MCLR. From the above, the main stages of strain CQ5-degrading MCLR were in stable phase and decline phase of strain growth. So Monod equation in the low substrate concentration was used to analyze the degradation process. And the dynamic relationship between strain CQ5-degrading MCLR and using it as the source of carbon and nitrogen to promote the self-growth of strain were proven.

Based on the data of the degradation test, the linear fitting of Eq. (6) $\left(V=-d S /(d t N)=K_{2} S\right)$ was done. The results were shown in Fig. 5.

It could be seen that the fitting equation between $V$ and $S$ was $V=0.342 S$. The correlation coefficient $\left(R^{2}\right)$ was 0.974 . When $K_{2}$ was $0.342, K_{2}$ was brought into Eq. (6) and transformed; the linkage relationship between MCLR mass concentration, cell density of strain CQ5, and the degradation rate of MCLR would be expressed by the following equation:

$-d S / d t=0.342 N S$

The integral was conducted on both sides of Eq. (11). Then the following equation would be obtained.

$S=14.12 e^{-0.342 N t}$

The dynamic relationship was derived between the degradation of MCLR by strain CQ5 and using it as a source of

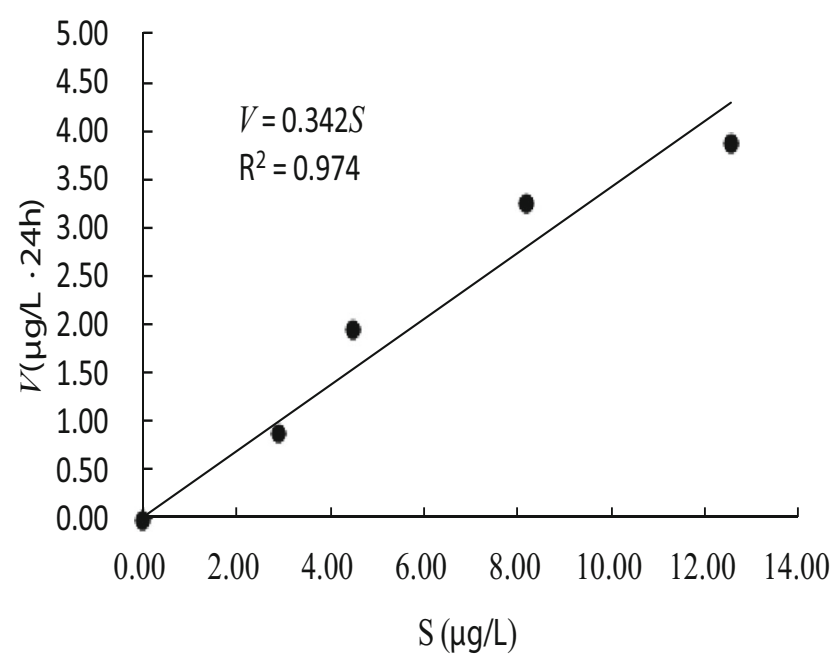

Fig. 5 The fitting curve of Monod between V and S. ( $V$, the specific degradation rate of MCLR; $S$, the concentration of MCLR)

carbon and nitrogen to promote its own growth. The comparisons between the first-order reaction kinetics equation $(\ln S=$ $2.64764-0.01537 t$ ) of describing the degradation of MCLR by strain CQ5 and Monod equation model $\left(S=14.12 e^{-0.342}\right.$ ${ }^{N t}, N=1.08$ ) were showed in Fig. 6. We could see that they were highly consistent. The maximum relative errors between the two models were less than $0.3 \%(0-0.19 \%)$. They both could simulate and predict the growth of strain CQ5 and the concentration of MCLR in the degradation system. But what calls for special attention is that the inoculated bacteria were from the bacterial solution cultivated for $24 \mathrm{~h}$ in the beef extract peptone medium. Strain CQ5 first used a small amount of peptone and nutrients with simple molecular structure from the crude extract after being transferred (Yang et al. 2009). For the meantime, strain CQ5 was in the process of adaptation of the exogenous and exotic substances from the new medium (Black 2012). Because peptone contains oligopeptide structure, which is similar to the molecular structure of MCs, strain CQ5 could utilize peptone and occasionally degrade less MCs to ensure normal growth. So the degradation rate of MCLR by strain CQ5 was slow at the beginning of inoculation. And that was probably why it can be obviously seen that when the concentration of MCLR was high at the initial stage of inoculation, there was a big difference between the measured values and predicted values of the two models (Fig. 6).

To sum up, the Monod model was suitable for the reaction system of degradation of MCLR by strain CQ5, which reflected the quantitative relationship among the MCLR concentration, cell density of strain CQ5, and MCLR degradation rate in the degradation process. As the purification process of activated sludge was divided into the initial removal of adsorption and the microbial metabolism (Lin 1986), analogies were that MCLR was decomposed by a series of biochemical reaction of strain CQ5 in crude extract. The decomposition products could be used as the supply of carbon and nitrogen 
Fig. 6 The forecast and comparisons between two models $(N=1.08, n=6, P>0.05)$. a First-order reaction kinetic model. b Monod equation model
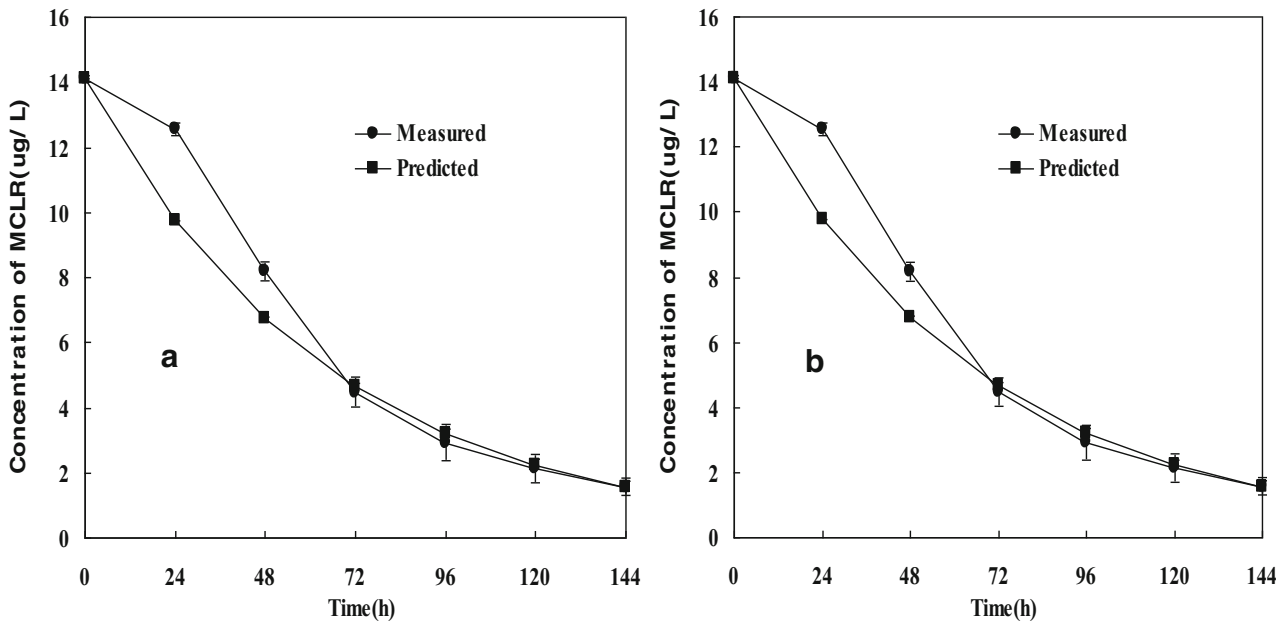

source for the growth strain CQ5. The single adsorption and condensation of polysaccharide slime layer covering on the cell surface might be not the main degradation mechanism. But if the degradation pathway and molecular mechanism of MCLR would be mastered, the degradation products should be further analyzed and more studies would be necessary to identify the degradation mechanism.

\section{Conclusion}

MCLR biodegradation was differentiated due to nutrient type and nutrient concentration. Lysinibacillus boronitolerans strain CQ5 capable of degrading MCLR was isolated and identified for the first time. This research found that the mass concentration of MCLR was reduced from 14.12 to $1.57 \mu \mathrm{g} / \mathrm{L}$ and the degradation rate of MCLR could reach $88.88 \%$ within $244 \mathrm{~h}$. When the initial $\mathrm{pH}$ value was 7 , the inoculation amount was 3\%, the screened Lysinibacillus boronitolerans strain CQ5 grew well in MSM, in which the MCLR crude extract was used as the carbon and nitrogen source.

The growth curve of strain CQ5 fitted with the Gompertz growth model $\left(N_{\mathrm{t}}=1.3119 * \exp (-0.1237 * \exp (-6.6341 t))\right.$, $\left.R^{2}>0.99\right)$. The process of MCLR degradation agreed with the first-order reaction kinetic equation $(\ln S=2.64764-$ $\left.0.01537 t, R^{2}>0.99\right)$. Strain CQ5 could degrade MCLR and use it as the source of carbon and nitrogen to promote its own growth $\left(S=14.12 e-0.342 N_{t}, N=1.08\right)$. During the degradation process, the linkage relationship between the mass concentration of MCLR, cell density of strain CQ5, and the degradation rate of MCLR could be expressed by the equation of revised Monod model $\left(V=0.342 S, R^{2}>0.97\right)$ under low substrate concentration, where $V_{\max } / K_{s}$ was 0.342 .

According to the results of dynamic analysis, it was referred that strain CQ5 decomposed and applied MCLR in crude extract mainly by a series of biochemical reactions. And it could use the decomposed products as the carbon and nitrogen sources for its growth. As a microbiological degradation method, strain CQ5 could promote the environmental fate of MCLR and have a certain practical value. This study would provide theoretical references for studying the mechanisms of MCLR biodegradation and promoting the engineering application of MCLR degradation bacteria.

Funding This work was supported by the National Natural Science Foundation (No. 41571471), the Jiangsu Science and Technology Support Project (No. BE2016653), and the Major Science and Technology Projects for Water Pollution Control and Treatment (2017ZX07202-003/004)

\section{Compliance with ethical standards}

Conflict of interest The authors declare that they have no conflict of interest.

Research involving human participants and/or animals This article does not contain any studies with human or animal subjects.

Informed consent Informed consent is not required in this study.

\section{References}

Black JG (2012) Microbiology principles and explorations, 8th edn. Wiley

Bourne DG, Riddles P, Jones GJ, Smith W, Blakeley RL (2010) Characterisation of a gene cluster involved in bacterial degradation of the cyanobacterial toxin microcystin LR. Environ Toxicol 16(6): $523-534$

Chen LM, Chai LH (2005) Mathematical model and mechanisms for biofilm wastewater treatment system. World J Microbiol Biotechnol 21(8-9):1455-1460

Chen LM, Chai LH (2006) A theoretical analysis on self-organized formation of microbial biofilms. PHYSICA A 370(2):793-807

Chen ZH, Dong XN, Shen HC, Chen D, Mao LQ, Zhang WY (2018) Study on screening and characteristics of algal-toxin degradation bacteria in reed the bottom mud of reed marshes from Lake Taihu. Ind Saf and Environ Prot (in Chinese) 44(5):29-32 
Dziga D, Wasylewski M, Wladyka B, Nybom S, Meriluoto J (2013) Microbial degradation of microcystins. Chem Res Toxicol 26(6): $841-852$

Edwards C, Graham D, Fowler N, Lawton LA (2008) Biodegradation of microcystins and nodularin in freshwaters. Chemosphere 73(8): $1315-1321$

Eleuterio L, Batista JR (2010) Biodegradation studies and sequencing of microcystin-LR degrading bacteria isolated from a drinking water biofilter and a fresh water lake. Toxicon 55(8):1434-1442

Guo XC, Lu SY, Xie P, Chen J, Liu XH (2016) Environmental exposure, toxicity and toxic mechanism of microcystins: a review. Asian J Ecotoxicol (in Chinese) 11(3):61-65

Hall T, Hart J, Croll B, Gregory R (2007) Laboratory-scale investigations of algal toxin removal by water treatment. Water Environ J 14(2): 143-149

Ho L, Hoefel D, Saint CP, Newcombe G (2007) Isolation and identification of a novel microcystin-degrading bacterium from a biological sand filter. Water Res 41(20):4685-4695

Ho L, Sawade E, Newcombe G (2012) Biological treatment options for cyanobacteria metabolite removal - a review. Water Res 46(5): $1536-1540$

Jia JP, He XQ, Jin Y (2009) Statistics (the 4th edition). China Renmin University Press

Lam AK, Fedorak PM, Prepas EE (1995) Biotransformation of the cyanobacterial hepatotoxin microcystin-LR, as determined by HPLC and protein phosphatase bioassay. Environ Sci Technol 29(1):242-246

Leyva-Díaz JC, Poyatos JM, Barghini P, Gorrasi S, Fenice M (2017) Kinetic modeling of Shewanella baltica KB30 growth on different substrates through respirometry. Microb Cell Factories 16:189-199

Li H, Pan G (2014) Enhanced and continued degradation of microcystins using microorganisms obtained through natural media. J Microbiol Methods 96(1):73-80

Li J, Peng L, Li J, Qiao Y (2014) Divergent responses of functional gene expression to various nutrient conditions during microcystin-LR biodegradation by Novosphingobium sp. THN1 strain. Bioresour Technol 156(2):335-341

Lin RC (1986) Research on the re-pollution of organic materials and the kinetics of organic material removal in biological wastewater treatment. J Tianjin Univ (in Chinese) 1:28-31

Manage PM, Edwards C, Singh BK, Lawton LA (2009) Isolation and identification of novel microcystin-degrading bacteria. Appl Environ Microbiol 75(21):6924-6928

Mu LN, Chen CW, Yu SZ, Liu JL, Wu YY, Zhu WC (2000) Investigation study on the content of microcystin in Tai Lake and methods for microcystin treatment. Chinese J Public Health (in Chinese) 16(9): 803-806

Oliveira CS, Ordaz A, Ferreira EC, Alves M, Thalasso F (2011) In situ pulse respirometric methods for the estimation of kinetic and stoichiometric parameters in aerobic microbial communities. Biochem Eng J (58-59):12-19

Ou YXR, Zhao QH, Wei YZ (2013) A preliminary exploration of dissolved oxygen based on FVCOM in Meiliang Bay, Lake Taihu and its influence mechanism. J Lake Sci (in Chinese) 25(4):478-488

Ramani A, Rein K, Shetty KG, Jayachandran K (2012) Microbial degradation of microcystin in Florida's freshwaters. Biodegradation 23(1):35-45

Rao LR, Li QY, Fan PC (2011) A method for extracting microcystin-LR from the cyanobacteria in Lake Taihu. CN102153635A

Rastogi RP, Sinha RP, Incharoensakdi A (2014) The cyanotoxinmicrocystins: current overview. Rev Environ Sci Biotechnol 13(2): 215-249
Rongsayamanont C, Limpiyakorn T, Law B, Khan E (2010) Relationship between respirometric activity and community of entrapped nitrifying bacteria: implications for partial nitrification. Enzym Microb Technol 46:229-236

Shimizu K, Maseda H, Okano K, Itayama T, Kawauchi Y, Chen R, Utsumi M, Zhang Z, Sugiura N (2011) How microcystindegrading bacteria express microcystin degradation activity. Lakes Reserv Res Manag 16(3):169-178

Song H, Reichwaldt ES, Ghadouani A (2014) Contribution of sediments in the removal of microcystin-LR from water. Toxicon 83(2):84-90

Spanjers H, Takacs I, Brouwer H (1999) Direct parameter extraction from respirograms for wastewater and biomass characterization. Water Sci Technol 39(4):137-145

Vareli K, Jaeger W, Touka A, Frillingos S, Briasoulis E, Sainis I (2013) Hepatotoxic seafood poisoning (HSP) due to microcystins: a threat from the ocean? Mar Drugs 11(8):2751-2768

Wang JB, Chai LH, Zhang Y (2013) Substrate-revised Monod equation-a new model on microorganism growth. Adv Microbiol 2:49-52

Wang FF, Wu Y, Gao Y, Chen ZL (2014) Biodegradation of microcystinLR by Burkholderia Vietnamiensis. Chin J Environ Eng 8(9):38373840

Wu YH, Kerr PG, Hu ZY, Yang LZ (2010) Removal of cyanobacterial bloom from a biopond-wetland system and the associated response of zoobenthic diversity. Bioresour Technol 101(11):3903-3908

Xie W, Wu J, Li YC, He XW, Du Y, Wang GY (2012) Screening and identification of microcystins degrading bacterial strain. J Biol (in Chinese) 29(6):35-38

Yan H, Wang HS, Liu XL, Yin CH, Xu QQ, Lu L, Ma WB (2014) Advances in the pathway and molecular mechanism for the biodegradation of microcystins. Environ Sci (in Chinese) 35(3):1205-1208

Yang JD, Yin YF, Hu LB, Zhou W, Ding HR, Shi ZQ, Yan SH (2009) Biodegradation of microcystins by indigenous mixed bacterial population JSM004. J Agro-Environ Sci (in Chinese) 28(8):1669-1672

Yang F, Zhou Y, Sun R, Wei H, Li Y, Yin L, Pu Y (2014) Biodegradation of microcystin-LR and-RR by a novel microcystin-degrading bacterium isolated from Lake Taihu. Biodegradation 25(3):447-457

You DJ, Chen XG, Xiang HY, Ou YL, Yang B (2014) Isolation, identification and characterization of a microcystin-degrading bacterium Paucibacter sp. strain CH. Environ Sci (in Chinese) 35(1):313-316

Zhang XH (2001) Principles and applications of higher environmental chemistry and microbiology. Chemical Industry Press, Beijing

Zhang J, Zheng Y, Li CY, Chai LH (2011) A confined DLA model of microbial biofilm in wastewater treatment systems. International Conference on Electrical and Control Engineering (ICECE), Yichang (16-18): 4234-4237

Zhao JB, Liu KY, Xue G, Cheng QY, Liu YN (2012) Research of screening and degradation characterstics of bacterium for the degradation of microcystins-LR. Chin J Environ Eng 6(2):671-674

Zhao S, Chen W, Jia YL, Zheng LL, Zuo YX, Song LR (2013) Characteristics of microcystin-degrading bacterium isolation from sedimentation ponds for the treatment of cyanobacterial blooms. Acta Hydrob Sinica 37(3):522-525

Zhu XY, Shen YT, Chen XG, Hu YOO, Xiang HY, Tao J, Ling Y (2016) Biodegradation mechanism of microcystin-LR by a novel isolate of rhizobium sp. TH and the evolutionary origin of the mlrA gene. Int Biodeterior Biodegrad 115:17-25

Publisher's note Springer Nature remains neutral with regard to jurisdictional claims in published maps and institutional affiliations. 